\title{
Information Theory of Quantum Systems with some hydrogenic applications
}

\author{
J.S. Dehesa ${ }^{*, \dagger}$, D. Manzano*,†, P. Sánchez-Moreno*,† and R.J. Yáñez ${ }^{* *, \dagger}$ \\ ${ }^{*}$ Dpto. de Física Atómica, Molecular y Nuclear, Universidad de Granada \\ ${ }^{\dagger}$ Instituto Carlos I de Física Teórica y Computacional, Universidad de Granada \\ ${ }^{*}$ Dpto. de Matemática Aplicada, Universidad de Granada
}

\begin{abstract}
The information-theoretic representation of quantum systems, which complements the familiar energy description of the density-functional and wave-function-based theories, is here discussed. According to it, the internal disorder of the quantum-mechanical non-relativistic systems can be quantified by various single (Fisher information, Shannon entropy) and composite (e.g. Cramér-Rao, LMC shape and Fisher-Shannon complexity) functionals of the Schrödinger probability density $\rho(\vec{r})$. First, we examine these concepts and its application to quantum systems with central potentials. Then, we calculate these measures for hydrogenic systems, emphasizing their predictive power for various physical phenomena. Finally, some recent open problems are pointed out.
\end{abstract}

Keywords: information theory, quantum mechanics, Fisher information, Shannon entropy, CramérRao complexity, LMC complexity, Fisher-Shannon complexity, hydrogenic systems

PACS: 89.70.Cf,03.67.-a, 31.15.-p

\section{INTRODUCTION}

The internal disorder of quantum-mechanical non-relativistic systems is conditioned by the spatial spreading of their Schrödinger single-particle probability density $\rho(\vec{r})$ to a great extent. To quantify it, various single and composite information-theoretic measures [1] have been proposed beyond the familiar ordinary moments $\left\langle r^{k}\right\rangle$ of $\rho(\vec{r})$,

$$
\left\langle r^{k}\right\rangle:=\int_{\Omega} r^{k} \rho(\vec{r}) d \vec{r} ; \quad k=0,1,2, \ldots
$$

and the statistical variance

$$
V[\rho]=\left\langle r^{2}\right\rangle-\langle r\rangle^{2}
$$

Among the single information-theoretic measures it is worth highlighting the Fisher information [2]

$$
F[\rho]:=\int_{\Omega} \frac{[\bar{\nabla} \rho(\vec{r})]^{2}}{\rho(\vec{r})} d \vec{r}
$$

and the Shannon entropy [3]

$$
S[\rho]:=\int_{\Omega} \rho(\vec{r}) \log \rho(\vec{r}) d \vec{r}
$$


and its generalization, the entropic moments

$$
W_{q}[\rho]:=\int_{\Omega}[\rho(\vec{r})]^{q} d \vec{r} ; \quad q \in \mathbb{R}
$$

or its modified forms known as Renyi and Tsallis entropies.

The (translationally invariant) Fisher information $F[\rho]$, contrary to the Shannon, Renyi and Tsallis entropies, is a local measure of spreading of the density $\rho(\vec{r})$ because it is a gradient functional of $\rho(\vec{r})$. The higher this quantity is, the more localized is the density, the smaller is the uncertainty and the higher is the accuracy in estimating the localization of the particle. In contrast, the Shannon, Renyi and Tsallis entropies are global measures of delocalization because they are logarithmic and power functionals of $\rho(\vec{r})$. They measure the total extent of the density in various complementary ways, without any reference to any specific point of the domain of definition of $\rho(\vec{r})$. The latter should be realized when comparing these three entropies with the variance $V[\rho]$.

These single information-theoretic measures have been shown to be very fertile in numerous scientific, technological and financial fields, particularly to identify, characterize and interpret numerous atomic and molecular phenomena such as e.g., correlation properties in atoms, spectral avoided crossings of atoms in external fields and the transition state and the other stationary points in chemical reactions.

Recently, some information-theoretic measures composed by two of the aforementioned single measures have been shown to be most appropriate to grasp the different facets of the internal disorder of quantum systems and to disentangle among their rich three-dimensional geometries. This is basically because (i) they are invariant under replication, translation and scaling transformations, and (ii) they have minimal values for both extreme cases: the completely ordered systems (e.g. a Dirac delta distribution and a perfect crystal in one and three dimensions) and the totally disordered systems (e.g., an uniform or highly flat distribution and an ideal gas in one and three dimensions). The latter property shows that these two-ingredient complexity measures quantify how easily a system may be modelled. These composite quantities are the LMC shape complexity $C_{\mathrm{LMC}}[\rho]$ which is defined [4] as

$$
C_{\mathrm{LMC}}[\rho]=D[\rho] \times \exp (S[\rho])
$$

the Fisher-Shannon complexity $C_{\mathrm{FS}}[\rho]$, defined in [5] by

$$
C_{\mathrm{FS}}[\rho]=F[\rho] \times J[\rho]
$$

and the Cramér-Rao complexity [5], given by

$$
C_{\mathrm{CR}}[\rho]=F[\rho] \times V[\rho]
$$

where $D[\rho]=W_{2}[\rho]$ is the second-order entropic moment, heretoforth called disequilibrium because it quantifies the departure of $\rho(\vec{r})$ from equilibrium, and $J[\rho]$ is the Shannon entropy power defined by

$$
J[\rho]=\frac{1}{2 \pi e} \exp \left(\frac{2}{3} S[\rho]\right)
$$


for the three-dimensional systems. We note that the LMC shape complexity measures the combined effect of the average height and the total spreading of the density, while the Fisher-Shannon complexity grasps the oscillatory nature of the density together with its total extent in the configuration space, and the Cramér-Rao quantity takes into account the gradient content of the density jointly with its concentration around the centroid. It is worth noting that these complexity measures are lower-bounded [6] as

$$
C_{\mathrm{LMC}}[\rho] \geq 1, \quad C_{\mathrm{FS}}[\rho] \geq 3 \quad \text { and } \quad C_{\mathrm{CR}}[\rho] \geq 9
$$

for general three-dimensional systems.

Here we first survey the information theory of quantum systems subject to central potentials and then we apply it to hydrogenic systems. Finally some applications to relativistic and multidimensional systems are shown to illustrate the predictive power of the theory.

\section{INFORMATION THEORETIC MEASURES OF CENTRAL POTENTIALS}

The quantum stationary states of a single-particle system in a spherically-symmetric potential $U(r)$ are known to be described by the wave functions

$$
\Psi_{n l m}(\vec{r})=R_{n l}(r) Y_{l m}(\theta, \phi)
$$

with the quantum numbers $n=1,2, \ldots, l=0,1,2, \ldots, n-1$, and $m=-l,-l+1 \ldots, l$. The angular part is given by the spherical harmonics

$$
Y_{l m}(\theta, \phi)=\frac{1}{\sqrt{2 \pi}} C_{l-m}^{(l+m)}(\cos \theta)(\sin \theta)^{m} e^{i m \phi}
$$

with $0 \leq \theta \leq \pi$ and $0 \leq \phi \leq 2 \pi$, and $C_{k}^{(\alpha)}(x)$ denotes the familiar Gegenbauer or ultraspherical polynomials. The radial part $R_{n l}(r)$ is given by an orthogonal hypergeometric function (i.e., it has the form $\omega^{1 / 2}(r) y_{n}(r)$, where $\left\{y_{n}(r)\right\}$ denotes a system of polynomials orthogonal with respect to the weight function $\omega(r))$ or any other special function of applied mathematics. Then the probability to find the particle between $\vec{r}$ and $\vec{r}+d \vec{r}$ is given by

$$
\rho_{n l m}(\vec{r}) d \vec{r}=\left|\Psi_{n l m}(\vec{r}, t)\right|^{2} d \vec{r}=D_{n l}(r) r^{2} d r \times \Pi_{l m}(\Omega) d \Omega,
$$

where the volume element $d \vec{r}=r^{2} d r \sin \theta d \theta d \phi \equiv r^{2} d r d \Omega$, and the radial and angular probability densities are given by

$$
D_{n l}(r)=\left|R_{n l}(r)\right|^{2}=\omega_{l}(r)\left[y_{n}(r)\right]^{2}
$$

and

$$
\Pi_{l m}(\Omega)=\left|Y_{l m}(\theta, \phi)\right|^{2}=\left[C_{l-m}^{l+m}(\cos \theta)\right]^{2}[\sin \theta]^{2 m}
$$

respectively. The former density gives the probability per radial interval to find the particle in $(r, r+d r)$, and the latter one describes the spatial profile of the system. 
The information theory allows us to calculate the intrinsic randomness (uncertainty) and the profile of the system by means of various information-theoretic measures (already defined) of the radial and angular probability densities, respectively. Beyond the variance, which is given by

$$
V\left[\rho_{n l m}\right]=\int_{0}^{\infty} r^{4} \omega_{l}(r)\left[y_{n}(r)\right]^{2} d r-\left|\int_{0}^{\infty} r^{3} \omega_{l}(r)\left[y_{n}(r)\right]^{2} d r\right|^{2},
$$

the Fisher information can be expressed by

$$
F\left[\rho_{n l m}\right]=F\left[y_{n}\right]+\left\langle r^{-2}\right\rangle F\left[Y_{l m}\right],
$$

where $\left\langle r^{-2}\right\rangle$ is equal to the norm of the orthogonal polynomials $y_{n}(r)$ involved in the wavefunction, and $F\left[y_{n}\right]$ and $F\left[Y_{l m}\right]$ are the Fisher functionals of the polynomial $y_{n}(r)$ and the spherical harmonics $Y_{l m}(\Omega)$, respectively. Moreover, the Shannon entropy turns out to be the sum of the entropic functionals $E\left[y_{n}\right]$ and $E\left[Y_{l m}\right]$, given by

$$
E\left[y_{n}\right]=-\int_{0}^{\infty} \omega_{l}(r) y_{n}^{2}(r) \log \left[\omega_{l}(r) y_{n}^{2}(r)\right] d r
$$

and

$$
E\left[Y_{l m}\right]=-\int_{0}^{\pi} \sin \theta d \theta \int_{0}^{2 \pi} d \phi\left|Y_{l m}(\theta, \phi)\right|^{2} \log \left|Y_{l m}(\theta, \phi)\right|^{2}
$$

The corresponding Cramér-Rao, Fisher-Shannon and LMC complexities can be subsequently obtained from their definitions (5)-(7), respectively, as products of two functionals of orthogonal polynomials of power, gradient or logarithmic type.

To go further in the determination of the spreading measures of the system we need to specify the analytic form of the central potential. This is done for hydrogenic systems, which have a Coulomb potential, in the following section.

\section{HYDROGENIC INFORMATION THEORY}

For a central potential of Coulombian form, i.e. $U(\vec{r})=-\frac{Z}{r}$, the quantum stationary states of a hydrogenic system have the energies $E_{n}=-\frac{Z^{2}}{2 n^{2}}$ and the probability density (12) $\rho_{n l m}(\vec{r})$ given by

$$
\rho_{n l m}(\vec{r})=R_{n l}^{2}(r)\left|Y_{l m}(\theta, \phi)\right|^{2}
$$

where

$$
R_{n l}^{2}(r)=\frac{4 Z^{3}}{n^{4}} \tilde{r}^{-1} \omega_{2 l+1}(\tilde{r})\left[\tilde{L}_{n-l-1}^{(2 l+1)}(\tilde{r})\right]^{2}
$$

with $\tilde{r}=\frac{2 Z}{n} r$ and $\omega_{\alpha}(\tilde{r})=\tilde{r}^{\alpha} e^{-\tilde{r}}$. Taking into account this fact and realizing that $y_{n}(r)$ are the Laguerre polynomials, we can obtain from Eqs. (15) and (16) the following values [9]

$$
V\left[\rho_{n l m}\right]=\frac{1}{4 Z^{3}}\left[n^{2}\left(n^{2}+2\right)-l^{2}(l+1)^{2}\right]
$$


and

$$
F\left[\rho_{n l m}\right]=\frac{4 Z^{3}}{n^{3}}(n-|m|)
$$

for the variance and Fisher information of any hydrogenic system, respectively. Note that they depend neither on $m$ (the variance) nor on $l$ (the Fisher information). Moreover, we can obtain that the Shannon entropy has the expression

$$
S\left[\rho_{n l m}\right]=B(n, l, m)-3 \log Z
$$

with

$$
B(n, l, m)=A_{1}(n, l, m)+\frac{1}{2 n} E_{1}\left[L_{n-l-1}^{(2 l+1)}\right]+E\left[C_{n-|m|}^{\left(|m|+\frac{1}{2}\right)}\right]
$$

being $A_{1}(n, l, m)$ a known expression, and where the entropic integrals

$$
E_{i}\left[y_{k}\right]:=-\frac{1}{\pi} \int_{a}^{b} x^{i} y_{k}^{2}(x) \log \left[y_{k}^{2}(x)\right] \omega(x) d x ; \quad i=0,1
$$

cannot be explicitly calculated except for circular $(n, l=m=n-1)$ and Rydberg $(n \gg 1)$ states. In particular, we obtain the values

$$
S\left[\rho_{100}\right]=3+\log \pi-3 \log Z
$$

and

$$
S\left[\rho_{n 00}\right]=6 \log n-\log 2+2 \log \pi+o(1)
$$

for the Shannon entropies of the ground state and the $(n s)$-Rydberg states, respectively.

Let us now calculate the complexity measures of the hydrogenic system [7] From Eqs. (5) and (21) jointly with the fact the disequilibrium $D[\rho]=Z^{3} A_{2}(n, l, m)$, being $A_{2}(n, l, m)$ a known expression, we obtain that the LMC shape complexity has the values

$$
C_{\mathrm{LMC}}\left[\rho_{n l m}\right]=A_{2}(n, l, m) e^{B_{2}(n, l, m)}
$$

where $A_{2}$ and $B_{2}$ are explicitly known functions of the three quantum numbers $(n, l, m)$. Similarly, from Eqs. (7), (19) and (20) we have the following value

$$
C_{\mathrm{CR}}\left[\rho_{n l m}\right]=\frac{n-|m|}{n^{3}}\left(n^{2}\left(n^{2}+2\right)-l^{2}(l+1)\right)^{2}
$$

for the Cramér-Rao complexity of the hydrogenic system. Moreover, Eqs. (6), (8), (16) and (21) yield the following value

$$
C_{\mathrm{FS}}\left[\rho_{n l m}\right]=\frac{4(n-|m|)}{n^{3}} \frac{1}{2 \pi e} e^{\frac{2}{3} B(n, l, m)}
$$

for the Fisher-Shannon complexity of the hydrogenic system.

We observe that the three complexity measures do not depend on the Coulombian strength, i.e. the nuclear charge $Z$. Moreover, the three complexities, according to Eqs. (22), (23) and (24), increase with increasing $n$ for $(l, m)$ fixed, and they decrease with 


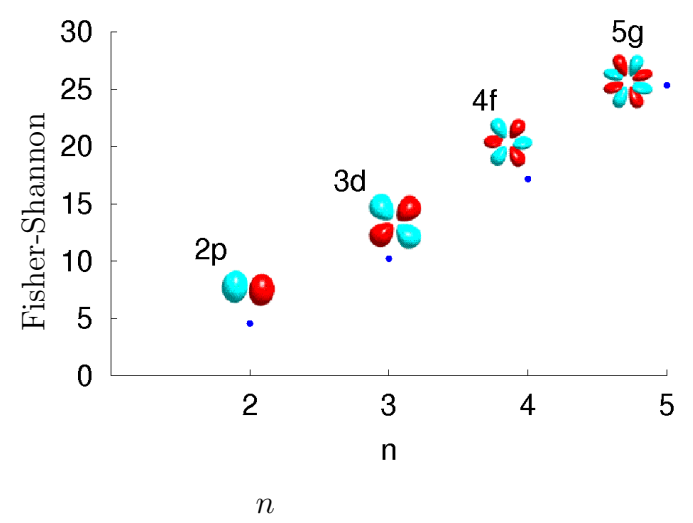

FIGURE 1. Fisher-Shannon complexity for quasi circular $(l=n-1)$ hydrogenic states in terms of the principal quantum number $n$.

increasing $l$ for $(n, m)$ fixed; the latter behaviour is also observed with $m$ for $(n, l)$ fixed. Finally, for completeness, we have plotted in Figure 1 the variation of the FisherShannon complexity (21) in terms of $n$ for various quasi-circular $(l=n-1)$ hydrogenic states. Therein, we observe the increasing value of the measure when the principal quantum number is increasing, clearly illustrating the idea of complexity in everyday life. Finally, let us also collect the values

$$
C_{\mathrm{CR}}\left[\rho_{100}\right]=3, \quad C_{\mathrm{LMC}}\left[\rho_{100}\right]=\frac{e^{3}}{8} \quad \text { and } \quad C_{\mathrm{FS}}\left[\rho_{100}\right]=\frac{2 e}{\pi^{1 / 3}}
$$

for the three complexity measures of the hydrogenic ground state.

\section{RELATIVISTIC AND DIMENSIONALITY APPLICATIONS}

Here we briefly consider some extensions of the previous information theory of hydrogenic systems to illustrate the predictive power of the complexity measures for relativistic and dimensionality phenomena. First, we show that the Fisher-Shannon complexity is a good quantitative indicator of the relativistic effects of hydrogenic systems in the Klein-Gordon framework. This is done by calculating this measure for pionic atoms with nuclear charge Z [8], taking care of the Lorentz invariance. In this case, the charge density has the expression

$$
\rho(\vec{r})=\frac{e}{m_{o} c^{2}}\left[\varepsilon-\frac{Z e^{2}}{r}\right]\left|\Psi_{n l m}(\vec{r})\right|^{2}
$$

where the pionic wavefunction $\Psi_{n l m}(\vec{r})$ is the physical solution of the Klein-Gordon equation

$$
\left(\varepsilon-\frac{Z e^{2}}{r}\right) \Psi(\vec{r})=\left(-\hbar c^{2} \vec{\nabla}^{2}+m_{0}^{2} c^{4}\right) \Psi(\vec{r})
$$

The Fisher-Shannon complexity (6) of the Lorentz-invariant density given by (25) and the duly normalized to the electronic charge $e$, has been calculated as a function of $Z$. The 


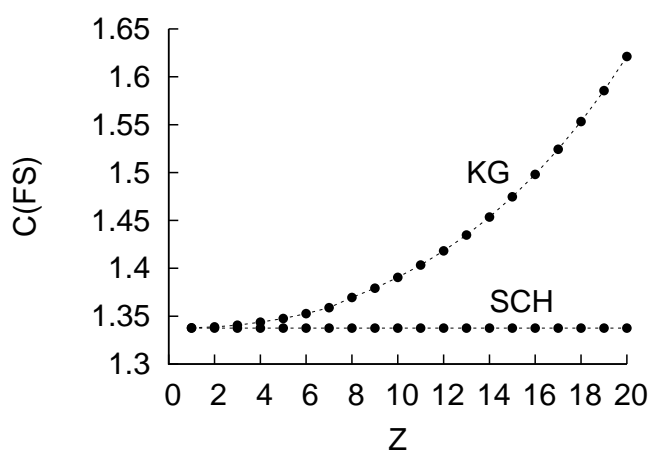

FIGURE 2. Fisher-Shannon complexity of the ground state of Klein Gordon and Schrödinger Coulomb equation in terms of the nuclear charge $Z$

results are plotted in Figure 2, where a comparison is also made with the Schrödinger or non-relativistic values of this measure. Therein, it is clear that the Klein-Gordon values of the Fisher-Shannon complexity do depend on the nuclear charge, contrary to the Schrödinger case (see also Eq. (24)). This is a clear indication that this complexity measure allows for a quantitative estimation of the relativistic effects on the internal disorder of the hydrogenic systems of pionic type. See Ref. [8] for further details.

Now, let us consider the LMC shape complexity (5) of the probability density

$$
\rho_{\mathrm{C} . \mathrm{S} .}(\vec{r})=\frac{2^{D+2-2 n} Z^{D} \prod_{j=1}^{D-2}\left(\sin \theta_{j}\right)^{2 n-2}}{\pi^{\frac{D-1}{2}}(2 n+D-3)^{D} \Gamma(n) \Gamma\left(n+\frac{D-1}{2}\right)} e^{-\frac{r}{\lambda}}\left(\frac{r}{\lambda}\right)^{2 n-2}
$$

corresponding to the circular states (i.e., $l=m=n-1$ ) of $D$-dimensional hydrogenic systems $[9,10]$, where $\vec{r}=\left(r, \theta_{1}, \theta_{2}, \ldots, \theta_{D-1}\right), \lambda=\frac{\eta}{2 Z}$, and $\eta=n+\frac{D-3}{2}$ is the principal hyperquantum number. The value has been recently obtained [10]

$$
\begin{aligned}
& C_{\mathrm{LMC}}\left[\rho_{\mathrm{C} . \mathrm{S} .}\right]=\frac{\Gamma\left(n-\frac{1}{2}\right) \Gamma\left(2 n+\frac{D-3}{2}\right)}{2^{2 n+D-2} \pi^{1 / 2} \Gamma\left(n+\frac{D-1}{2}\right)} \\
& \exp \left\{2 n+D-2-(n-1)\left[\psi(n)+\psi\left(n+\frac{D-1}{2}\right)\right]\right\},
\end{aligned}
$$

(where $\psi(x)=\Gamma^{\prime}(x) / \Gamma(x)$ is the digamma function) for the position LMC complexity of a $D$-dimensional hydrogenic system in an arbitrary circular state. The corresponding dimensionality dependence of this measure is plotted in Figure 3 for the ground state $(n=1)$ and the excited circular states with $n=2$ and 3. Therein, we note a parabolic growth for all states when the dimensionality is increasing, being always greater than unity in accordance with Eq. (9). In addition, we observe that the minimum value of this complexity measure is $(e / 2)^{2}=1.847$, occurring for $D=2$. 


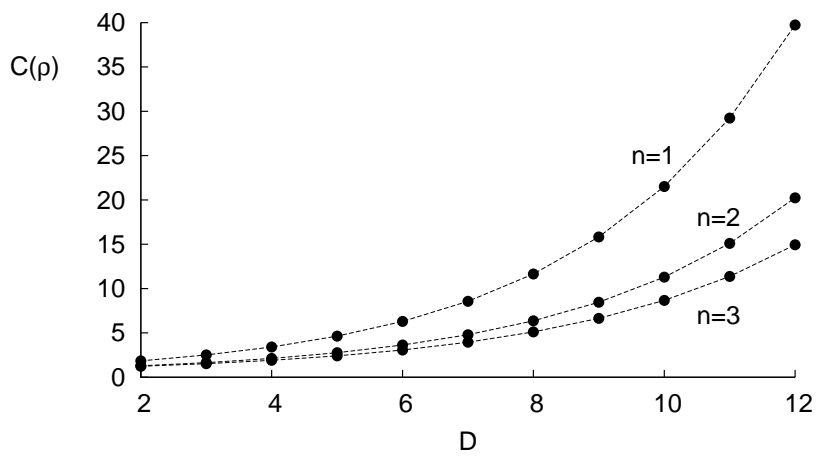

FIGURE 3. LMC complexity of hydrogenic circular states with $n=1,2$ and 3 in terms of the dimension $D$

\section{CONCLUSION AND OPEN PROBLEMS}

In summary we have surveyed various aspects of the emerging information theory of quantum systems with central potentials, emphasizing the results obtained for hydrogenic states. Furthermore, we have shown the predictive power of the complexity measures for the relativistic and dimensionality effects of some ground and excited hydrogenic states.

Let us finally mention a few open problems in this field. First, to study the analytical and asymptotical properties of the entropic moments $W_{q}[\rho]$ for both central and Coulombian potentials. Second, to mutually compare the various direct spreading measures in the sense of Hall [11] for hydrogenic states. Third, to analyze the behaviour of the complexity measures of $D$-dimensional systems in the presence of external magnetic and electric fields. Fourth, to examine the information-theoretic measures of many-electron atoms moving on a $D$-dimensional hypersphere.

\section{ACKNOWLEDGMENTS}

We are very grateful for the partial financial support of the grants FIS2008-02380, FQM2445, FQM-4643 and FQM-207 of the Junta de Andalucia and Ministerio de Ciencia e Innovacion, Spain, EU.

\section{REFERENCES}

1. J.S. Dehesa, S. López-Rosa and D. Manzano, in K.D. Sen (ed.) "Statistical Complexities: Application to Electronic Structure" (Springer-Verlag, Berlin, 2010)

2. B.R. Frieden, Science from Fisher Information. (Cambridge Univ. Press, 2004)

3. C.E. Shannon and W. Weaver, The Mathematical Theory of Communication (University of Illinois Press, 1949)

4. R.G. Catalan, J. Garay and R. López-Ruiz, Phys. Rev E 66, 011102 (2002)

5. J.C. Angulo, J. Antolín and R.O. Esquivel, in K.D. Sen (ed.) "Statistical Complexities: Application to Electronic Structure" (Springer-Verlag, Berlin, 2010)

6. S. López-Rosa, J.C. Angulo and J. Antolín, Physica A 388, 2081-2091 (2009) 
7. J.S. Dehesa, S. López-Rosa and D. Manzano, Eur. Phys. J. D 55 539-549 (2009).

8. D. Manzano, S. López-Rosa and J.S. Dehesa, Europhys. Lett. 90, 48001 (2010)

9. J.S. Dehesa, S. López-Rosa, A. Martínez-Finkelshtein and R.J. Yáñez, Int. J. Quant. Chem. 110, 1529$1548(2010)$

10. S. López-Rosa, D. Manzano and J.S. Dehesa, Physica A 388, 3273-3281 (2009)

11. M.J.W. Hall, Phys. Rev. A 58, 2602 (1999) 